\title{
Effects of lentiviral vector-mediated shRNA silencing of TGFß1 on the expression of Col1a1 in rat hepatic stellate cells
}

\author{
GAN-PING BAI ${ }^{1 *}$, GUO-HE YAN ${ }^{2 *}$, GUO-JIAN WANG ${ }^{1}$, PING WAN $^{1}$, XIAO-FEI LIU ${ }^{1}$, \\ HENG WANG ${ }^{1}$ and RONG-HUA ZHANG ${ }^{1}$ \\ ${ }^{1}$ Department of Integrated Chinese and Western Medicine, Southwest Hospital; ${ }^{2}$ Institute of Combined Injuries, \\ The State Key Laboratory of Trauma, Burns and Combined Injury, College of Preventive Medicine, \\ The Third Military Medical University, Chongqing 400038, P.R. China
}

Received August 9, 2016; Accepted May 11, 2017

DOI: $10.3892 / \mathrm{mmr} .2017 .6945$

\begin{abstract}
The present study aimed to construct a lentiviral RNA interference (RNAi) vector targeting the transforming growth factor $\beta 1$ (TGF $\beta 1$ ) gene of rats, in order to examine its effect on silencing of the TGF $\beta 1$ gene and on the expression of collagen type $1 \alpha 1$ (Colla1) in HSC-T6 rat hepatic stellate cells. Three RNAi sites of the TGF $\beta 1$ gene were selected according to its CDs sequence. Three pairs of small interfering RNA (siRNA) of these RNAi sites were synthesized and then transfected into HSC-T6 cells, respectively, to confirm the optimal siRNA sequence via reverse transcription-polymerase chain reaction analysis. Subsequently, shRNA targeting the sequence of the optimal siRNA was designed, synthesized and annealed to form a double-stranded structure. The annealed oligonucleotide fragment was cloned into pGreenPuro plasmids to establish the pGreenPuro/TGF $\beta 1$ shRNA lentiviral vector, which was then transfected into 293T cells, following identification by restriction enzyme digestion and sequencing for the production of lentiviral particles exhibiting high reactivity. These particles were used to infect HSC-T6 cells, following which the expression of GFP in the transfected cells was observed under an inverted microscope. The effects on TGF $\beta 1$ gene silencing and the expression levels of Colla1 were detected at the mRNA and protein levels. The results provided confirmation of the optimal siRNA sequence. Enzyme digestion and sequencing verified successful construction of the pGreenPuro/TGF $\beta 1$ shRNA
\end{abstract}

Correspondence to: Dr Rong-Hua Zhang, Department of Integrated Chinese and Western Medicine, Southwest Hospital, The Third Military Medical University, 29 Gaotanyan Avenue, Shapingba, Chongqing 400038, P.R. China

E-mail: zhrhlggg@163.com

*Contributed equally

Key words: transforming growth factor $\beta 1$ gene, collagen type $1 \alpha 1$ gene, RNA interference, lentiviral vector lentiviral vector. This lentiviral vector effectively silenced the TGF $\beta 1$ gene in the HSC-T6 cells, and inhibited the expression of Colla1 at the mRNA and protein levels. Taken together, the lentiviral RNAi vector targeting the TGF $\beta 1$ gene of rats was successfully constructed, which effectively silenced the TGF $\beta 1$ gene of the HSC-T6 cells and inhibited the expression of Colla1.

\section{Introduction}

Liver cirrhosis is a common disease clinically and is life-threatening in humans. It features an imbalance in the synthesis and degradation of extracellular matrix (ECM), primarily collagen, leading to metabolic disorders of the whole liver and a series of severe clinical complications. Hepatic fibrosis constitutes an inevitable pathological stage in the transformation from multiple chronic liver diseases to cirrhosis, in which the activation and proliferation of hepatic stellate cells (HSCs) is pivotal (1). HSC activation and the following abnormal expression of ECM are essential to the onset of liver cirrhosis. Transforming growth factor $\beta 1$ (TGF $\beta 1$ ) functions as a crucial factor in activating HSCs and promoting its expression of ECM. The TGF $\beta 1$ signal transduction pathway is important in regulating the expression of ECM in HSCs (2). Therefore, TGF $\beta 1$ is one of the significant targets in the prevention and treatment of hepatic fibrosis.

In the present study, the optimal small interfering RNA (siRNA) sequence targeting the TGF $\beta 1$ gene was first confirmed, and the corresponding pGreenPuro/TGF $\beta 1$ short hairpin (sh)RNA lentiviral vector was constructed and transfected into HSC-T6 cells in vitro to observe the lentiviral vector-mediated effect of shRNA on TGF $\beta 1$ gene silencing and on the expression of collagen type $1 \alpha 1$ (Colla1) of the ECM, which was anticipated to provide an experimental basis for the prevention and treatment of hepatic fibrosis.

\section{Materials and methods}

Materials. The HSC-T6 cell line was obtained from Shanghai Aiyan Biotech (Shanghai, China); synthesis of the oligonucleotide sequences of the three RNA interference (RNAi) sites and the corresponding controls was performed by Guangzhou 
RiboBio Co., Ltd. (Guangzhou, China); the gel extraction kit and plasmid extraction kit were from Roche Diagnostics (Basel Switzerland); restriction enzymes, T4 DNA ligase, Taq polymerase and the reverse transcription-polymerase chain reaction (RT-PCR) kit were from Takara Bio, Inc. (Otsu, Japan); the pGreenPuro lentiviral vector was from Shanghai Innovation Biotechnology Co., Ltd. (Shanghai, China); Lipofectamine ${ }^{\circledR} 2000$ was from Invitrogen; Thermo Fisher Scientific, Inc. (Waltham, MA, USA); anti-TGF $\beta 1$ antibody and anti-Colla1 antibody were from Merck Millipore (Darmstadt, Germany); DNA oligosynthesis of the optimal siRNA sequence, and the primer synthesis and sequencing were performed by Shanghai Generay Biotech Co., Ltd. (Shanghai, China).

Designing and screening of the optimal siRNA site of TGF $\beta 1$. A total of three RNAi sites of the TGF $\beta 1$ gene of rats were selected, according to its CDs sequence (site 1, GTCAACTGT GGAGCAACAC; site 2, GCACCATCCATGACATGAA; site 3, CCGCAACAACGCAATCTAT). The corresponding siRNAs of the three sites were synthesized respectively: Site 1 sense, 5'-GUCAACUGUGGAGCA ACACdTdT-3' and antisense, 5'-dTdTCAGUUGACACCUCGUUGUG-3'; site 2 sense, 5'-GCACCAUCCAUGACAUGAAdTdT-3' and antisense, 5'-dTdTCGUGGUAGGUACUGUACUU-3'; site 3 sense, 5'-CCGCAACAACGCAAUCUAUdTdT-3' and antisense, 5'-dTdTGGCGUUGUUGCGUUAGAUA-3'. The three pairs of siRNAs were transfected into HSC-T6 cells, respectively, according to the manufacturer's protocol.

The detection primers of TGF $\beta 1$ (NM_021578.2) and GAPDH (AF106860.2) of rats were designed based on their respective gene sequences. The upstream and downstream primers of TGF $\beta 1$ were 5'-ACTACGCCAAAGAAGTCA CCC-3' and 5'-TGAGCACTGAAGCGAAAGC-3', respectively, and the upstream and downstream primers of GAPDH were 5'-TCTACTGGCGTCTTCA-3' and 5'-TGAGCCCTT CCACGAT-3', respectively. Total RNA of HSC-T6 cells transfected with each siRNA was extracted using TRIzol reagent (Invitrogen; Thermo Fisher Scientific, Inc.) according to manufacturer's protocol. With the total RNA of HSC-T6 cells transfected with each siRNA, respectively, and with template and non-transfected HSC-T6 cells as controls, RT-PCR analysis was performed to confirm the optimal siRNA site among the three siRNA sites.

Construction and identification of the TGF $\beta 1$ shRNA lentiviral vector. The shRNA targeting the sequence of the optimal siRNA was designed and synthesized as follows: Sense, 5'-GATCCGTCAACTGTGGAGCAACACCTTCCTGTCAG AGTGTTGCTCCACAGTTGACTTTTTG-3' and antisense, 5'-AATTCAAAAAGTCAACTGTGGAGCAACACTCTGA CAGGAAGGTGTTGCTCCACAGTTGACG-3'; BamH and EcoR digestion sites were introduced into the positive-sense and antisense strands, respectively, and annealed to form a double-stranded structure. The annealed oligonucleotide fragment was cloned into the pGreenPuro plasmid to establish the pGreenPuro/TGF $\beta 1$ shRNA lentiviral vector, which was then transfected into competent cells. The positive recombinant plasmid DNA was identified by restriction enzyme digestion and sequencing.
Packaging and titer of TGF 1 shRNA lentiviral recombinants. Trypsin was used to digest the healthy 293T cells (Type Culture Collection of Chinese Academy of Sciences, Shanghai, China), which were in the logarithmic phase, $24 \mathrm{~h}$ prior to transfection, and the cells were then diluted with DMEM high-glucose medium (DMEM; HyClone; GE Healthcare, Chicago, IL, USA) containing 10\% FBS (catalog no. F2442; Sigma-Aldrich; Merck KGaA, Darmstadt, Germany). and, inoculated into a 6 -well plate at $5 \times 10^{5}$ cells/well for incubation at $37^{\circ} \mathrm{C}, 5 \% \mathrm{CO}_{2}$ and saturated humidity. When the culture reached a 70-80\% density, Lipofectamine ${ }^{\circledR} 2000$ gene transfection reagent was used to mediate transfection of the pGreenPuro/TGF $\beta 1$ shRNA lentiviral plasmid DNA with the right sequence into $293 \mathrm{~T}$ cells. The medium was renewed at $8 \mathrm{~h}$ and cultivation was continued until $48 \mathrm{~h}$ under the same conditions. An inverted fluorescence microscope was used to observe the expression of GFP and capture images.

The incubation was continued until $72 \mathrm{~h}$, when the supernatant of each culture was collected for centrifugation at $4,000 \times \mathrm{g}$ and $4^{\circ} \mathrm{C}$ for $10 \mathrm{~min}$ to remove cell debris. A filter $(0.45 \mu \mathrm{m})$ was used for further filtration. Following concentration, the supernatant was stored in aliquots at $-70^{\circ} \mathrm{C}$.

Titers were detected and calculated using the double dilution method with the following formula: Viral titer $=(\mathrm{P} \times \mathrm{N} / 100$ $\mathrm{x}$ V) $\mathrm{x} 1 / \mathrm{DF}$; in which $\mathrm{P}$ represents the GFP-positive cell count, $\mathrm{N}$ represents $10^{5}, \mathrm{~V}$ represents the volume of viral diluent and DF represents the dilution ratio.

Transfection of HSC-T6 cells with the pGreenPuro/TGF $\beta 1$ shRNA lentiviral plasmid. Trypsin was used to digest the healthy $293 \mathrm{~T}$ cells in the logarithmic phase $24 \mathrm{~h}$ prior to transfection, following which the cells were then diluted with DMEM high-glucose medium containing 10\% FBS and inoculated into a 6 -well plate at $5 \times 10^{5}$ cells/well for incubation at $37^{\circ} \mathrm{C}, 5 \% \mathrm{CO}_{2}$ and saturated humidity. When the culture reached a $70-80 \%$ density, the pGreenPuro/TGF $\beta 1$ shRNA lentiviral solution was added to the medium for transfection at a ratio of 1:50, with HSC-T6 cells transfected with empty vector or non-transfected cells as controls. An inverted fluorescence microscope was used to observe the expression of GFP and capture images 24-48 h later.

Effects of the pGreenPuro/TGF $\beta 1$ shRNA lentiviral vector on the protein expression of TGF $\beta 1$ and Collal in HSC-T6 cells. At $48 \mathrm{~h}$ post-transfection with the pGreenPuro/TGF $\beta 1$ shRNA lentiviral vector, the total protein was extracted by western and IP cell lysis solution (Beyotime Institute of Biotechnology, Haimen, China) according to the manufacturer's protocol from the HSC-T6 cells. The protein concentration was measured using a bicinchoninic acid assay kit (Beyotime Institute of Biotechnology), and $20 \mu \mathrm{g}$ protein was separated by $10 \%$ SDS-PAGE and transferred onto nitrocellulose membranes. Membranes were soaked in blocking buffer containing a TGF $\beta 1$ rabbit polyclonal antibody (1:600; catalog no. sc-146) or a Col1a1 mouse monoclonal antibody (1:500; catalog no. sc-59772) (both from Santa Cruz Biotechnology, Inc., Dallas, TX, USA), respectively, overnight at $4^{\circ} \mathrm{C}$. Subsequently, HRP-labeled mouse anti-rabbit secondary antibody (1:400; catalog no. A0208) and rabbit anti-mouse secondary antibody (1:400; catalog no. A0216) (both from Beyotime Institute of 
Biotechnology, Inc.) were added, respectively, and incubated at room temperature for $1.5 \mathrm{~h}$. Chemiluminescence substrate was added for co-culture for $\sim 7 \mathrm{~min}$ at $25^{\circ} \mathrm{C}$. Images of the results were then captured using GADPH $\left(\mathrm{MW}, 3.7 \times 10^{4}\right)$ as the internal reference.

Effects of TGF $\beta 1$ shRNA on the gene expression of TGF $\beta 1$ and COL1 in HSC-T6 cells. The specific detection primers for rat Colla1 (NM_053304.1) were designed based on its gene sequence: Upstream 5'-TGATGTATGCTTGATCTG TAT-3' and downstream 5'-CGGGGACCCATTGGACCT GAA-3'. The specific detection primers of rat TGF $\beta 1$ and GAPDH were as described above. Total RNA of HSC-T6 cells transfected with TGF $\beta 1$ shRNA was used as the template, and the gene expression of TGF $\beta 1$ and COL1 were detected using RT-PCR analysis, with empty vector-transfected cells treated as controls and GADPH gene as the internal reference. A total of $1.2 \mu \mathrm{g}$ RNA from each sample was transcribed into cDNA using the PrimeScript ${ }^{\mathrm{TM}}$ RT kit (Takara Bio, Inc.) according to the manufacturer's protocol. Reverse transcription was performed under the following conditions: $30^{\circ} \mathrm{C}$ for $10 \mathrm{~min}$ and $42^{\circ} \mathrm{C}$ for $30 \mathrm{~min}$ to synthesize the first chain of cDNA; $99^{\circ} \mathrm{C}$ for $5 \mathrm{~min}$ to inactivate reverse transcriptase; cooling at $5^{\circ} \mathrm{C}$ for $5 \mathrm{~min}$, one circle. The RT product $(12 \mu \mathrm{l})$ was diluted in water to a total volume of $50 \mu \mathrm{l}$. cDNA was then amplified by PCR with primers specific to the target TGF $\beta 1$ and Collal sequence. PCR was performed under the following conditions: Predenaturation at $95^{\circ} \mathrm{C}$ for $5 \mathrm{~min}$, denaturation at $94^{\circ} \mathrm{C}$ for $45 \mathrm{sec}$, annealing at $57^{\circ} \mathrm{C}$ for $1 \mathrm{~min}$, extension at $72^{\circ} \mathrm{C}$ for $1 \mathrm{~min}$ ( 28 circles) and final extension at $72^{\circ} \mathrm{C}$ for $5 \mathrm{~min}$. The PCR products $(5 \mu \mathrm{l})$ were subjected to electrophoresis and confirmed using GoldView ${ }^{\mathrm{TM}}$ staining.

\section{Results}

Screening of the optimal siRNA site in TGF 1 1. RT-PCR analysis was used to confirm the optimal siRNA site among the three sites. Site 1 was identified as optimal, with the sequence GTCAACTGTGGAGCAACAC (Fig. 1).

Construction and identification of the TGF $\beta 1$ shRNA lentiviral vector. The recombinant $\mathrm{pGreenPuro/TGF} \beta 1$ shRNA lentiviral vector was digested using $B a m \mathrm{H} 1$ and $E c o$ RI to produce a 55 bp shRNA fragment, shown by electrophoresis (Fig. 2). Sequencing verified successful construction of the recombinant pGreenPuro/TGF $\beta 1$ shRNA lentiviral vector carrying TGF $\beta 1$ shRNA.

Packaging and titer of TGF $\beta 1$ shRNA lentiviral recombinants. Lipofectamine ${ }^{\circledR} 2000$ gene transfection reagent was used to mediate pGreenPuro/TGF $\beta 1$ shRNA lentiviral plasmid DNA transfection of 293T cells (Fig. 3A). Following incubation for $48 \mathrm{~h}$, marked GFP expression was observed under the fluorescence microscope (Fig. 3B). The culture showed a viral titer of $7 \times 10^{7} \mathrm{TU} / \mathrm{ml}$ via the double dilution method.

Transfection of HSC-T6 cells with the pGreenPuro/TGF $\beta 1$ shRNA lentiviral plasmid. No expression of GFP was detected in the non-transfected HSC-T6 cells (Fig. 4A), whereas marked expression of GFP was observed under the fluorescence

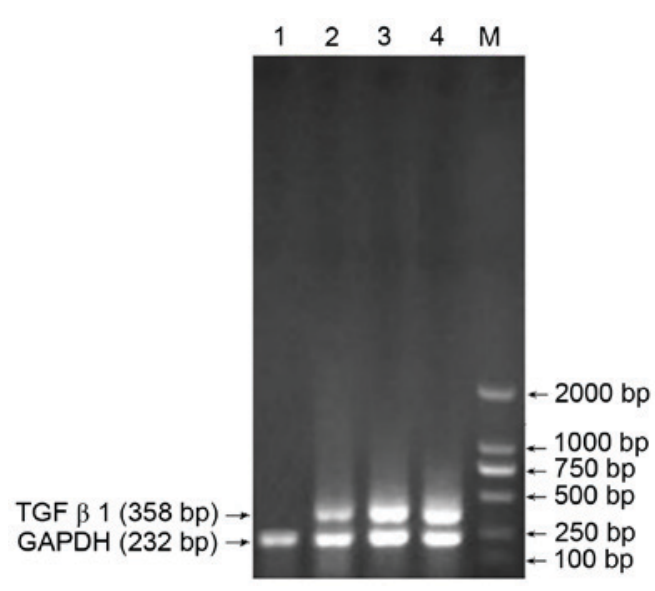

Figure 1. Identification of three target RNA interference sites of TGF $\beta 1$ in HSC-T6 cells. Results of electrophoresis are shown. 1, target; 2, target; 3 , target; 4, comparison; M, DNA standard (DL 2000). TGF $\beta 1$, transforming growth factor $\beta 1$.

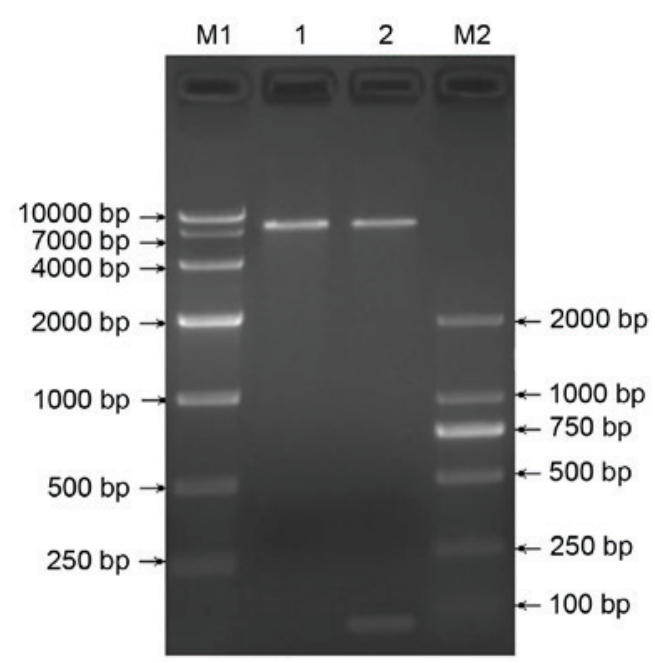

Figure 2. Construction and identification of the TGF $\beta 1$ shRNA lentiviral vector. Slow enzyme digestion of recombinant viral vector pGreenPuro/TGF $\beta 1$ shRNA was performed. M1 and M2, DNA standards; 1, pGreenPuro/TGF $\beta 1$ shRNA; 2, BamHI and EcoRI digested pGreenPuro/TGF $\beta 1$ shRNA. TGF $\beta 1$, transforming growth factor $\beta 1$; shRNA, short hairpin RNA.

microscope in the HSC-T6 cells transfected with the pGreenPuro/TGF $\beta 1$ shRNA lentiviral plasmid (Fig. 4B).

Effects of the pGreenPuro/TGF $\beta 1$ shRNA lentiviral vector on the protein expression of TGF $\beta 1$ and Collal in HSC-T6 cells. The results of the western blot analysis showed that the HSC-T6 cells transfected with pGreenPuro/TGF $\beta 1$ shRNA lentiviral plasmid produced faintly-stained anti-TGF $\beta 1$ and anti-Colla1 bands at MW $1.25 \times 10^{4}$ and $1.35 \times 10^{5}$, respectively, whereas the empty vector-transfected HSC-T6 cells and non-transfected HSC-T6 cells produced intense staining of bands at the two sites (Fig. 5), which indicated that silencing of the TGF $\beta 1$ gene in HSC-T6 cells at the RNA level effectively inhibited the expression of Colla1.

Effects of TGF $\beta 1$ shRNA on the gene expression of TGF $\beta 1$ and Collal in HSC-T6 cells. With the total RNA of the 

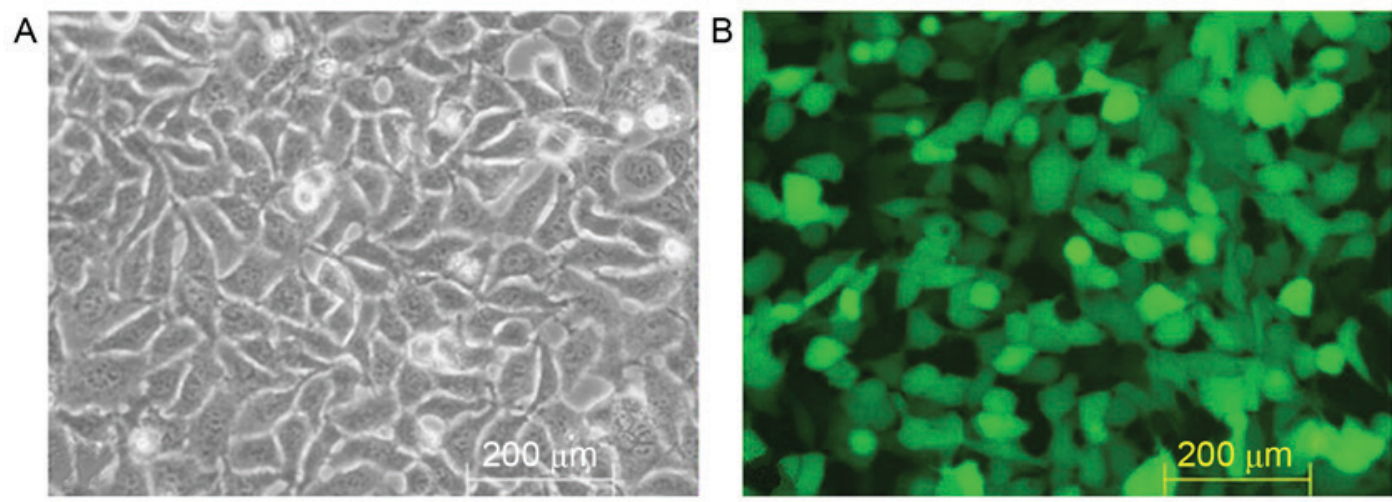

Figure 3. Packaging and titer of TGF $\beta 1$ shRNA lentiviral recombinants. Under a normal fluorescence microscope (A) 293T cells did not express GFP, whereas (B) 293 T cells packaged with recombinant lentivirus pGreenPuro/TGF 1 shRNA expressed GFP. Scale bar, $200 \mu \mathrm{m}$. TGF $\beta 1$, transforming growth factor $\beta 1$; shRNA, short hairpin RNA.

A

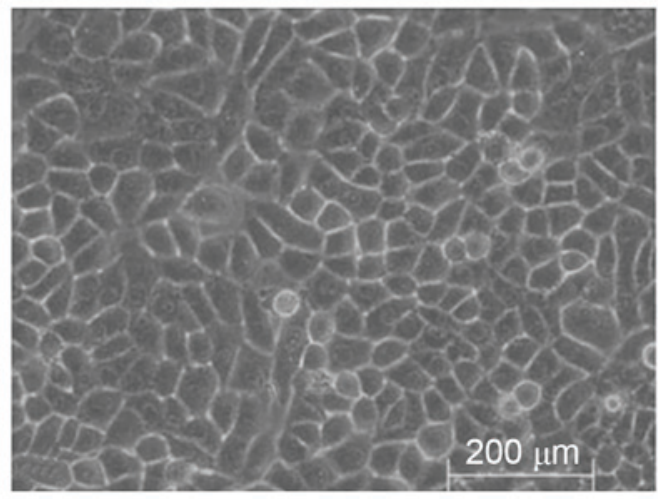

B

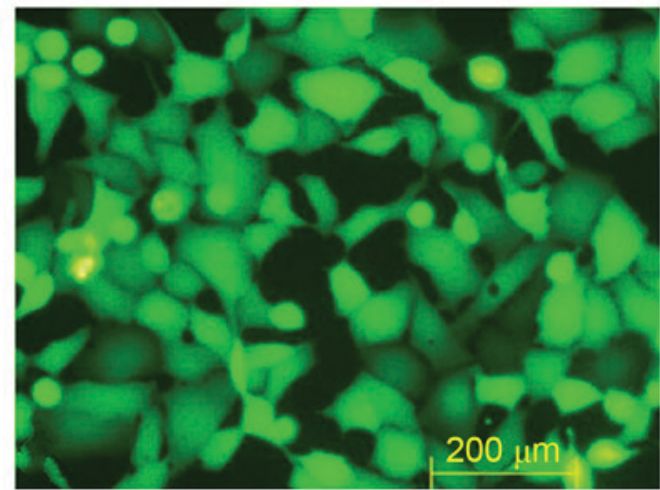

Figure 4. Transfection of HSC-T6 cells with the pGreenPuro/TGF $\beta 1$ shRNA lentiviral plasmid. Under the microscope (A) uninfected HSC-T6 cells did not express GFP. (B) pGreenPuro/TGF $\beta 1$ shRNA lentivirus infected-HSC-T6 cells expressed GFP. Scale bar, $200 \mu \mathrm{m}$. TGF $\beta 1$, transforming growth factor $\beta 1$; shRNA, short hairpin RNA.

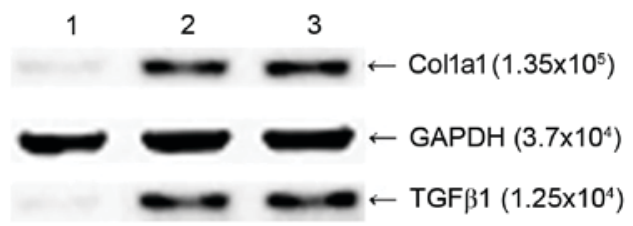

Figure 5. TGF $\beta 1$ shRNA affects HSC-T6 cell expression of TGF $\beta 1$ and Col1a1. Electrophoresis output shows protein expression levels of TGF $\beta 1$ and Colla1. 1, TGF 31 shRNA-infected HSC-T6 cells; 2, empty vector-infected HSC-T6 cells; 3, uninfected HSC-T6 cells. TGF $\beta 1$, transforming growth factor $\beta 1$; Colla1, collagen type I $\alpha 1$; shRNA, short hairpin RNA.

HSC-T6 cells $48 \mathrm{~h}$ following transfection with the pGreenPuro/TGF $\beta 1$ shRNA lentiviral plasmid as a template, the RT-PCR assay showed no expression of TGF $\beta 1$ and comparatively mild expression of Colla1 at the mRNA level. With total RNA from the HSC-T6 cells at $48 \mathrm{~h}$ post-transfection with the empty plasmid or non-transfected HSC-T6 cells as templates, the results of the RT-PCR assay revealed high expression levels of TGF $\beta 1$ and Colla1 at the mRNA level (Fig. 6). These results indicated that the recombinant pGreenPuro/TGF $\beta 1$ shRNA lentiviral plasmid effectively silenced the TGF $\beta 1$ gene and downregulated the expression of Colla1 at the mRNA level.

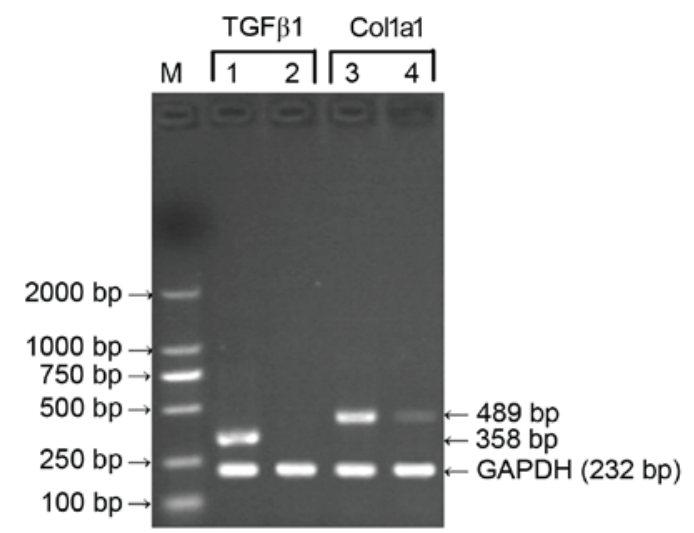

Figure 6. TGF $\beta 1$ shRNA affects HSC-T6 cell gene expression of TGF $\beta 1$ and Colla1. M, DNA standard (DL2000); 1 and 3, empty vector-infected HSC-T6 cells; 2 and 4, TGF $\beta 1$ shRNA-infected HSC-T6 cells. TGF $\beta 1$, transforming growth factor $\beta 1$; Col1a1, collagen type I $\alpha 1$; shRNA, short hairpin RNA.

\section{Discussion}

Hepatic fibrosis represents a pathological stage in which abnormal hyperplasia of intrahepatic connective tissues is manifested during multiple chronic liver injury repair processes. It features an imbalance in the generation and 
degradation of ECM, which leads to the excessive deposition of ECM, predominantly Colla1, and finally results in decreased hepatic function.

HSCs are located in Disse's interspace, close to liver sinusoidal endothelial cells and hepatocytes. There are only a small number of HSCs in the normal liver, representing 5-8\% of all hepatocytes. HSCs bind with vitamin A lipid droplets, and engage in vitamin A metabolism and ECM synthesis (3), which contribute to the excessive deposition of ECM, particularly under pathological conditions. Activated HSCs have a leading role in the process of hepatic fibrosis, where they multiply in quantity, exhibit phenotype changes and producing excessive ECM.

Derived from rat primary hepatic stellate cells infected by the T antigen of SV40, the HSC-T6 cell line is an active form of HSCs, which can express and secrete collagen matrix, and is an ideal model for investigating hepatic fibrosis (4).

Myofibroblasts produce abundant collagen fibers during acute or chronic liver injury, primarily types I and III, with the level of Colla1 markedly increased. Type I and III collagen accumulate excessively in Disse's space and occupies the interspace of liver sinusoidal endothelial cells, which promotes the onset and development of hepatic fibrosis. Collal constitutes a primary stage of ECM in the case of hepatic fibrosis and cirrhosis, and its expression level can be used as an important indicator to reflect the degree of hepatic fibrosis. High levels of Colla1 synthesis is central in the onset and development of hepatic fibrosis. TGF $\beta$, which is composed of six subtypes: TGF $\beta 1$, TGF $\beta 2$, TGF $\beta 3$, TGF $\beta 1 \beta 2$, TGF $\beta 4$ and TGF $\beta 5$, is essential to the physiological and pathological status of the liver (5), and TGF $\beta 1$ has the highest contribution to hepatic fibrosis among the multiple coefficient cytokines (6,7). TGF $\beta 1$ regulates ECM deposition resulting from tissue injury-mediated physiological response and also pathological reactions. TGF $\beta 1$ is predominantly expressed by liver sinusoidal endothelial cells and Kupffer cells, and mild expression is observed in HSCs in the normal liver, which is markedly upregulated in HSCs in the case of liver injury. The changes in TGF $\beta 1$ and Colla1 showed a consistent trend during hepatic fibrosis, as TGF $\beta 1$ directly or indirectly activated HSCs to increase the generation of Colla1-dominating ECM and decrease its degeneration. The synthesis of ECM, particularly Col1a1, can be reduced via inhibiting the generation of TGF $\beta 1$ and inhibiting its secretion and amplification processes. Therefore, TGF $\beta 1$ functions as an important initiating factor of hepatic fibrosis. It is clear that silencing TGF $\beta 1$ can be used as an important strategy in the prevention and treatment of hepatic fibrosis. Inhibiting the expression or biological activities of TGF $\beta 1$ via antisense oligonucleotides or drugs can reduce the generation of ECM and the deposition of fibrous protein $(8,9)$, although this is ineffective. However, the increase of RNAi techniques provides novel strategies for reducing ECM generation and fibrous protein deposition via gene silencing.

RNAi refers to sequence-specific posttranscriptional gene silencing in which endogenous or exogenous double-stranded RNA combines with and degrades intracellular mRNA with the homologous sequence (10). The RNAi technique has high specificity, efficiency and stability (11), and is a useful tool with which gene function and interrelation among upstream and downstream molecules in signal transduction systems are investigated. However, this technique is limited by the fact that the transfection of short-chain RNA can only be sustained for a few days in mammalian cells, whereas lentiviral vector techniques can realize longer term gene expression inhibition, which is more appropriate for in vivo experiments (12).

Therefore, the present study selected three RNAi sites in the rat TGF $\beta 1$ gene according to its CDs sequence, and the optimal siRNA sequence was confirmed using RT-PCR analysis. Subsequently, shRNA targeting the sequence of the optimal siRNA was designed, synthesized and cloned into the human pGreenPuro plasmid to establish the pGreenPuro/TGF $\beta 1$ shRNA lentiviral vector, and HSC-T6 cells were effectively transfected with this recombinant lentiviral vector. RT-PCR and western blot analyses confirmed that the pGreenPuro/TGF $\beta 1$ shRNA lentiviral vector effectively mediated the expression of TGF $\beta 1$ in the HSC-T6 cells silenced by TGF $\beta 1$ shRNA, and simultaneously downregulated the expression of Colla1. These results indicated that TGF $\beta 1$ shRNA downregulated the activating signal of HSCs to reduce the generation of Colla1-dominating ECM of HSCs, to alleviate or inhibit hepatic fibrosis. Therefore, TGF $\beta 1$ shRNA is among the novel strategies for the prevention and treatment of hepatic fibrosis.

The present study was limited by the fact that the expression of Colla1 was detected only at the mRNA and protein levels following TGF- $\beta$ interference. The secretion of Collal and changes in other components of the ECM, including collagen type III, also require consideration in the overall situation, requiring further investigation.

\section{Acknowledgements}

This study was supported by the National Natural Science Foundation (grant no. 81273918), the Project of Traditional Chinese Medicine Science and Technology of Chongqing (grant no. 2012-2-63) and the Specific Project of Traditional Chinese Medicine of Chinese PLA, Chongqing, China (grant no. 2010ZYZ231).

\section{References}

1. Mann DA and Marra F: Fibrogenic signalling in hepatic stellate cells. J Hepatol 52: 949-950, 2010.

2. Xu L, Zheng N, He Q, Li R, Zhang K and Liang T: Puerarin, isolated from Pueraria lobata (Willd.), protects against hepatotoxicity via specific inhibition of the TGF- $\beta 1 / \mathrm{Smad}$ signaling pathway, thereby leading to anti-fibrotic effect. Phytomedicine 20: 1172-1179, 2013.

3. Atzori L, Poli G and Perra A: Hepatic stellate cell: A star cell in the live. Int J Biochem Cell Biol 41: 1639-1642, 2009.

4. Vogel S, Piantedosi R, Frank J, Lalazar A, Rockey DC, Friedman SL and Blaner WS: An immortalized rat liver stellate cell line (HSC-T6): A new cell model for the study of retinoid metabolism in vitro. J Lipid Res 41: 882-893, 2000.

5. Baghy K, Iozzo RV and Kovalszky I: Decorin-TGF $\beta$ axis in hepatic fibrosis and cirrhosis. J Histochem Cytochem 60: 262-268, 2012.

6. Inagaki Y, Higashiyama R and Higashi K: Novel anti-fibrotic modalities for liver fibrosis: Molecular targeting and regenerative medicine in fibrosis therapy. J Gastroenterol Hepatol 27 (Suppl 2): S85-S88, 2012.

7. Gressner OA, Rizk MS, Kovalenko E, Weiskirchen R and Gressner AM: Changing the pathogenetic roadmap of liver fibrosis? Where did it start; where will it go? J Gastroenterol Hepatol 23: 1024-1035, 2008. 
8. Kajdaniuk D, Marek B, Borgiel-Marek H and Kos-Kudła B Transforming growth factor $\beta 1$ (TGFbeta1) in physiology and pathology. Endokrynol Pol 64: 384-396, 2013.

9. Ieronimakis N, Hays AL, Janebodin K, Mahoney WM Jr, Duffield JS, Majesky MW and Reyes M: Coronary adventitial cells are linked to perivascular cardiac fibrosis via TGF $\beta 1$ signaling in the mdx mouse model of Duchenne muscular dystrophy. J Mol Cell Cardiol 63: 122-134, 2013.

10. Ashihara E, Kawata E and Maekawa T: Future prospect of RNA interference for cancer therapies. Curr Drug Targets 11: $345-360,2010$
11. Chen J and Xie J: Progress on RNAi-based molecular medicines. Int J Nanomedicine 7: 3971-3980, 2012.

12. Zeng Z, Zhang $C$ and Chen J: Lentivirus-mediated RNA interference of DC-STAMP expression inhibits the fusion and resorptive activity of human osteoclasts. J Bone Miner Metab 31: 409-416, 2013. 